University of Wollongong

Research Online

Australian Institute for Innovative Materials -

Papers

Australian Institute for Innovative Materials

$1-1-2016$

Point defects in epitaxial silicene on $\mathrm{Ag}(111)$ surfaces

Hongsheng Liu

Dalian University of Technology

Haifeng Feng

University of Wollongong, hf533@uowmail.edu.au

Yi Du

University of Wollongong, ydu@uow.edu.au

Jian Chen

Chinese Academy Of Sciences

Ke-Hui Wu

Chinese Academy Of Sciences

See next page for additional authors

Follow this and additional works at: https://ro.uow.edu.au/aiimpapers

Part of the Engineering Commons, and the Physical Sciences and Mathematics Commons

Research Online is the open access institutional repository for the University of Wollongong. For further information contact the UOW Library: research-pubs@uow.edu.au 


\title{
Point defects in epitaxial silicene on Ag(111) surfaces
}

\author{
Abstract \\ Silicene, a counterpart of graphene, has achieved rapid development due to its exotic electronic \\ properties and excellent compatibility with the mature silicon-based semiconductor technology. Its low \\ room-temperature mobility of $\sim 100 \mathrm{~cm} 2 \mathrm{~V}-1 \mathrm{~s}-1$, however, inhibits device applications such as in field- \\ effect transistors. Generally, defects and grain boundaries would act as scattering centers and thus \\ reduce the carrier mobility. In this paper, the morphologies of various point defects in epitaxial silicene on \\ $\mathrm{Ag}(111)$ surfaces have been systematically investigated using first-principles calculations combined with \\ experimental scanning tunneling microscope (STM) observations. The STM signatures for various \\ defects in epitaxial silicene on $\mathrm{Ag}(111)$ surface are identified. In particular, the formation energies of point \\ defects in $\mathrm{Ag}$ (111)-supported silicene sheets show an interesting dependence on the superstructures, \\ which, in turn, may have implications for controlling the defect density during the synthesis of silicene. \\ Through estimating the concentrations of various point defects in different silicene superstructures, the \\ mystery of the defective appearance of $\$$ \sqrt $\{13\} \backslash$ times $\backslash$ sqrt $\{13\} \$$ and $\$ 2 \backslash$ sqrt $\{3\} \backslash$ times $2 \backslash$ sqrt $\{3\} \$$ \\ silicene in experiments is revealed, and $4 \times 4$ silicene sheet is thought to be the most suitable structure \\ for future device applications. \\ Disciplines \\ Engineering | Physical Sciences and Mathematics \\ Publication Details \\ Liu, H., Feng, H., Du, Y., Chen, J., Wu, K. \& Zhao, J. (2016). Point defects in epitaxial silicene on Ag(111) \\ surfaces. 2D Materials, 3 (2), 025034-1-025034-12.

\section{Authors} \\ Hongsheng Liu, Haifeng Feng, Yi Du, Jian Chen, Ke-Hui Wu, and Jijun Zhao
}




\title{
Point defects in epitaxial silicene on Ag(111) surfaces
}

\author{
Hongsheng $\mathrm{Liu}^{1}$, Haifeng Feng ${ }^{2}$, Yi Du ${ }^{2 *}$ Jian Chen ${ }^{3}$, Kehui Wu ${ }^{3}$ Jijun Zhao ${ }^{1,4 *}$ \\ ${ }^{1}$ Key Laboratory of Materials Modification by Laser, Ion and Electron Beams (Dalian University \\ of Technology), Ministry of Education, Dalian 116024, China \\ ${ }^{2}$ Institute for Superconducting and Electronic Materials (ISEM), University of Wollongong, \\ Wollongong, New South Wales 2525, Australia \\ ${ }^{3}$ Beijing National Laboratory for Condensed Matter Physics and Institute of Physics, Chinese \\ Academy of Sciences, Beijing 100190, China \\ ${ }^{4}$ Beijing Computational Science Research Center, Beijing 100084, China
}

\begin{abstract}
Silicene, a counterpart of graphene, has achieved rapid development due to its exotic electronic properties and excellent compatibility with the mature silicon-based semiconductor technology. Its low room-temperature mobility of $\sim 100 \mathrm{~cm}^{2} \cdot \mathrm{V}^{-1} \mathrm{~s}^{-1}$, however, inhibits device applications such as in field-effect transistors. Generally, defects and grain boundaries would act as scattering centers and thus reduce the carrier mobility. In this paper, the morphologies of various point defects in epitaxial silicene on $\operatorname{Ag}(111)$ surfaces have been systematically investigated using first-principles calculations combined with experimental scanning tunneling microscope (STM) observations. The STM signatures for various defects in epitaxial silicene on $\mathrm{Ag}(111)$ surface are identified. In particular, the formation energies of point defects in $\mathrm{Ag}(111)$-supported silicene sheets show an interesting dependence on the superstructures, which, in turn, may have implications for controlling the defect density during the synthesis of silicene. Through estimating the concentrations of various point defects in different silicene superstructures, the mystery of the defective appearance of $\sqrt{13} \times \sqrt{13}$ and $2 \sqrt{3} \times 2 \sqrt{3}$ silicene in experiments is revealed, and $4 \times 4$ silicene sheet is thought to be the most suitable structure for future device applications.
\end{abstract}




\section{Introduction}

Silicene, a monolayer of silicon atoms arranged in a honeycomb lattice, has attracted great attention in recent years [1-6]. In contrast to flat graphene, silicene possesses a low-buckled structure with a buckled height of about $0.44 \AA$ [7-8]. Nevertheless, silicene exhibits excellent electronic properties [7-15] similar to those of graphene [16-20]. Its band structure exhibits a linear dispersion and shows characteristic massless Dirac fermions with the Fermi velocity of $10^{5}$ $10^{6} \mathrm{~ms}^{-1}[7,12,15]$. Due to strong spin-orbit coupling (SOC), the quantum spin Hall effect may be observed in silicene in an experimentally accessible temperature regime [13]. A tunable band gap can be opened up to about $4 \mathrm{eV}$ in silicene by applying a perpendicular electric field [21-22] and by hydrogenation [23-26], halogenation [27-28], and oxidation [29-30]. Owing to these excellent properties and easy integration into the current Si-based semiconductor technology, silicene holds great promise for future applications in nanoelectronic devices.

To date, silicene with various superstructures, including $(4 \times 4),(\sqrt{13} \times \sqrt{13}) \mathrm{R} 13.9^{\circ},(\sqrt{7} \times \sqrt{7}$ R19.1 $1^{\circ},(2 \sqrt{3} \times 2 \sqrt{3}) \mathrm{R} 30^{\circ}$ with respect to $\operatorname{Ag}(111)$ [31-36], and $(\sqrt{3} \times \sqrt{3})$ with respect to silicene $1 \times 1$ lattice [11, 31, 37], have been fabricated on Ag(111) surfaces. Very recently, a silicene field-effect transistor (FET) was successfully fabricated following a growth-transfer-fabrication process, in which the silicene device was encapsulated by delamination with native electrodes [38]. Nevertheless, the measured carrier mobility at room temperature was only about $100 \mathrm{~cm}^{2} \mathrm{~V}^{-1} \mathrm{~s}^{-1}$, which is three orders of magnitude lower than that of perfect free-standing silicene $\left(2.6 \times 10^{5}\right.$ $\mathrm{cm}^{2} \mathrm{~V}^{-1} \mathrm{~s}^{-1}$ for electrons and $2.2 \times 10^{5} \mathrm{~cm}^{2} \mathrm{~V}^{-1} \mathrm{~s}^{-1}$ for holes) [12] and even lower than that of a monolayer $\mathrm{MoS}_{2}$ FET $\left(\sim 200 \mathrm{~cm}^{2} \mathrm{~V}^{-1} \mathrm{~s}^{-1}\right)$ [39]. Such a low mobility may be attributed to structural defects in the silicene. Generally, defects are inevitable in two-dimensional (2D) materials and have a significant impact on their physical properties. Therefore, a deep understanding of defects is highly desirable before fabrication of large-scale high-quality silicene layers for device applications. Recently, several typical point defects, including Stone-Wales (SW) rotation, single and double vacancies (SVs and DVs), and silicon adatoms in freestanding silicene have been systematically investigated using density functional theory (DFT) calculations, focusing on the geometries, energetics, and effects on electronic properties [40-50]. It was found that the SWs and DVs may induce small gaps in silicene, while the SV defect leads to a semimetallic-to-metallic transition in silicene. Using the molecular dynamics finite element method with Tersoff potential, 
Le reported that a single defect would significantly reduce the fracture strength of a silicene sheet [44]. Moreover, vacancy defects can reduce the thermal conductivity and the thermal stability of silicene [45-46].

Despite the above efforts, a comprehensive understanding of point defects in epitaxial silicene is still lacking. In particular, most previous calculations considered only freestanding silicene. Despite that many previous experiments have observed defective features in the atomic structures of the Ag(111)-supported silicene samples [34-35, 51-57], there is no direct experimental identification on the point defects in epitaxial silicene. To address this critical issue, here, we present a systematical exploration of various types of point defects, including SW, SV, DV, and adatom in epitaxial silicene on $\mathrm{Ag}(111)$ surface using scanning tunneling microscopy combined with first-principles calculations. The agreement between the simulated STM images and the measured ones clarifies the atomic structures of point defects in epitaxial silicene. The formation energies and possible diffusion behavior of defects in two common silicene superstructures are compared and their implications for the defect density in experimentally synthesized silicene sheets are discussed.

\section{Results and discussion}

Four kinds of point defects in epitaxial silicene, including SW, SV, DV, and silicon adatom, were considered in this work. In addition, possible imperfections of the Ag(111) surface, including SV and silver adatom, were also explored. In order to distinguish defects in silicene layer and on Ag(111) surface, the defects in silicene are denoted as Si-SW, Si-SV, Si-DV and Si-ad for SW, SV, DV and silicon adatom defects, respectively, while the defects in $\mathrm{Ag}(111)$ surface are denoted as Ag-SV and Ag-ad for single vacancy and silver adatom defects, respectively. Different configurations for one kind of defect are denoted by the numbers at end of their names. For example, Si-SW-1 and Si-SW-2 denote two different structures of Stone-Wales defects in silicene layer. Since the $4 \times 4, \sqrt{13} \times \sqrt{13}$ and $2 \sqrt{3} \times 2 \sqrt{3}$ superstructures are the most commonly observed structures of epitaxial silicene on $\mathrm{Ag}(111)$ surfaces [31-36, 53], we focus on these three superstructures in this paper. In freestanding silicene, there are two sets of sub-lattices; thus, the configurations of point vacancies (SW, SV, DV) are not dependent on local positions in a silicene sheet. The buckling of Si atoms in a silicene sheet would be rearranged, however, when the 
silicene is deposited on an $\mathrm{Ag}(111)$ surface. Therefore, the structure of a point defect in epitaxial silicene on the Ag surface becomes position dependent. For example, different morphologies for a Si-SV within one unit cell of a given superstructure can occur, depending on whether the missing Si atom comes from the buckled-up atoms or the buckled-down atoms. In view of this fact, several possible structures for each kind of point defect have been taken into account for different silicene superstructures. To characterize the thermodynamic stability of a point defect in silicene, we define its formation energy $E_{\text {form }}$ as:

$$
E_{\text {form }}=E_{\text {defect }}+N \times \mu_{\text {Si }}-E_{\text {perfect }}
$$

where $E_{\text {defect }}$ and $E_{\text {perfect }}$ are the energies for defective and perfect silicene on Ag supercells, respectively. $N$ is the number of missing atoms in the defective silicene on Ag supercell. For Si-ad, $N$ is set to be $-1 . \mu_{\mathrm{Si}}$ is the energy of one silicon atom in its bulk phase.

\subsection{Structures of various defects in $4 \times 4$ silicene}

Atomic structures of some representative point defects in the $4 \times 4$ silicene superstructure are shown in Figure 1. The Si-SW defect in a $4 \times 4$ silicene sheet, formed by a Si-Si bond rotation of $90^{\circ}$, has the same configuration as in freestanding silicene [40, 47, 49], which is composed of two pentagons and two heptagons (Figure 1a). The Si-SW defect in different positions results in a similar structure regardless of small differences in the detailed buckled pattern and formation energy, as depicted in Figure S1(a-d) in the Supporting Information. It is noteworthy that the formation energy for Si-SW in epitaxial silicene (1.354-1.544 eV) is lower than in previous predictions for freestanding silicene (2.09 eV [40], $1.64 \mathrm{eV}$ [47], and $1.82 \mathrm{eV}$ [49]), owing to the passivation effect of the Ag substrate [58]. 

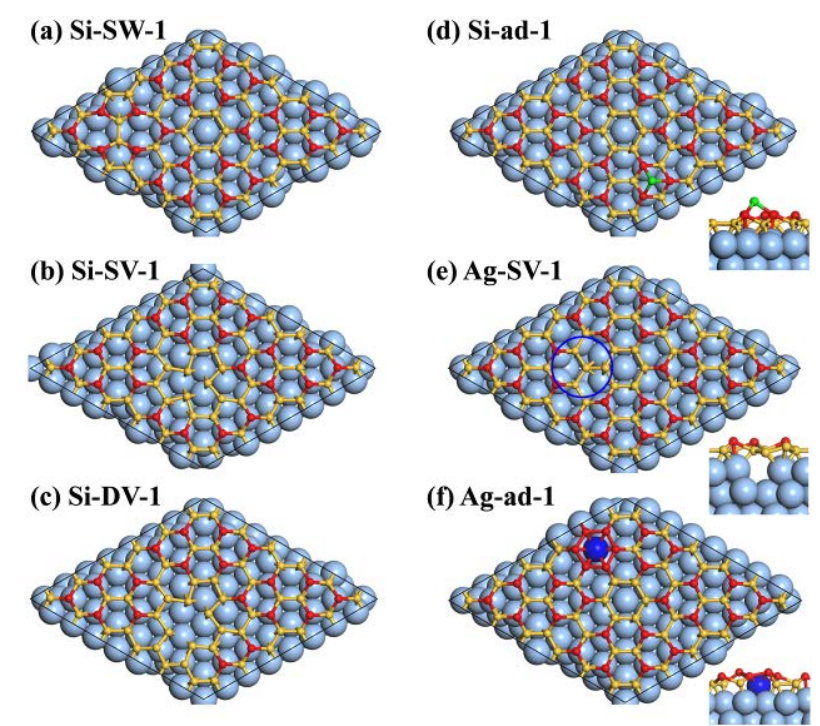

Figure 1. Atomic structures of several representative point defects in silicene and Ag substrate with a $4 \times 4$ silicene on $\mathrm{Ag}(111)$ superstructure. The small pictures in (d), (e), and (f) are the corresponding local side views around the defects. The sky-blue, dark-blue, yellow, red, and green balls represent $\mathrm{Ag}$ atoms, $\mathrm{Ag}$ adatoms, buckled-down Si atoms, buckled-up Si atoms, and Si adatoms, respectively. The dark blue circle indicates the position of the Ag vacancy. The black rhombus indicates the simulation supercell.

For Si-SV in a $4 \times 4$ silicene sheet, the configuration with three two-coordination silicon atoms (Si-SV-1, Figure 1b) is more energetically favorable $\left(E_{\text {form }}=0.731 \mathrm{eV}\right)$ than the reconstructed configuration (Si-SV-2, Figure S1e) proposed previously for freestanding silicene $\left(E_{\text {form }}=1.059\right.$ eV) [40]. For Si-SV-2, existence of two pentagons induces a four-coordination central Si atom (Figure S1e). This is different from SV in graphene, in which a carbon SV would induce one pentagon and one nonagon [59]. Again, the difference between freestanding and epitaxial silicene sheets can be ascribed to the passivation effect of the Ag surface. In freestanding silicene, the three dangling bonds created by one Si-SV would significantly increase the energy. Hence, local structural reconstruction is needed to remove the dangling bonds. In epitaxial silicene, however, passivation by the Ag surface would stabilize the dangling bonds created by the defect, which is a common feature for silicene superstructures on metal substrates [58, 60-62]. As a representative example, Si-DV-1 in $4 \times 4$ silicene is displayed in Figure 1c, which is similar to the DV-1 $(5|8| 5)$ proposed in our previous study [40]. Also note that Si-SV and Si-DV defects would severely 
influence the local buckling pattern of silicene, e.g. by reducing the height of the buckled-up silicon atoms by $\sim 0.7 \AA$. For a $\mathrm{Si}$ adatom on $4 \times 4$ silicene, the preferred adsorption site is the hollow site of a hexagonal ring (Figure 1d), in which three silicon atoms are buckled up and the other three silicon atoms are buckled down, forming three Si-Si bonds with length of $\sim 2.5 \AA$. A number of other metastable configurations for Si-SV, Si-DV, and Si-ad have been considered, and the details can be found in Supporting Information S1.

In this work, the effect on the morphology of epitaxial silicene due to possible imperfection of the Ag(111) surface, including Ag-SV and Ag-ad, was also taken into account. Combining DFT calculations and experimental STM observations, Satta and co-workers recently suggested that during the growth of silicene on $\mathrm{Ag}(111)$ surface, some Si atoms may penetrate the first $\mathrm{Ag}(111)$ layer and expel the Ag atoms [63]. Therefore, Ag-SV and Ag-ad may exist during the growth of silicene even though the prepared $\mathrm{Ag}(111)$ surface is flat enough. For Ag-SV-1 (Figure 1e), the missing Ag atom is right underneath the original buckled-up Si atom. After geometry relaxation, the original buckled-up Si atom moves down and becomes a buckled-down atom; while the other Si atoms are affected little by the Ag vacancy, with maximum displacement from their original locations by $0.05 \AA$. For Ag-ad, taking Ag-ad-1 (Figure 1f) as representative, one Ag adatom is located right underneath a hollow site of the silicene honeycomb lattice, lifting up the six silicon atoms in the hexagonal ring by about $1.33 \AA$. Several other types of Ag adatoms are displayed in Figure S3 of the Supporting Information. 


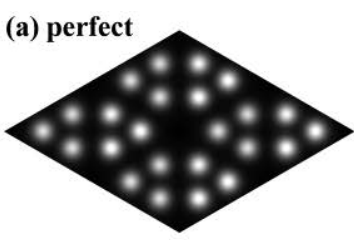

(b) Si-SV-1

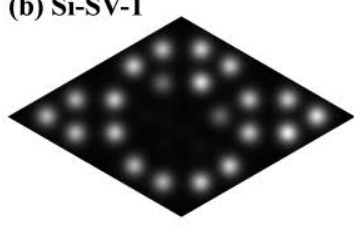

(c) $\mathrm{Si}-\mathrm{DV}-1$

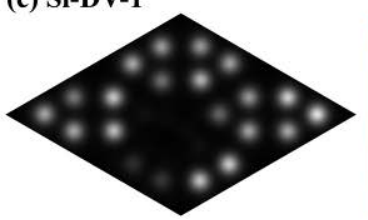

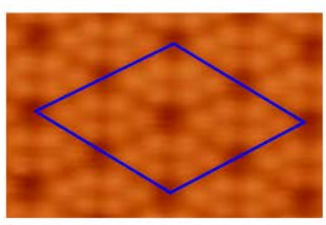
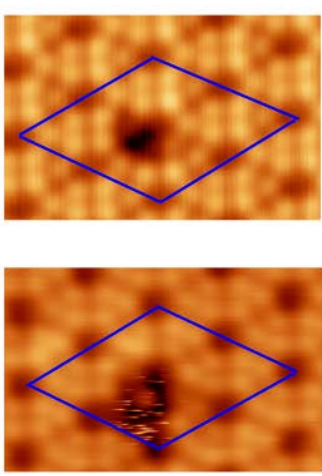

(d) Si-ad-1

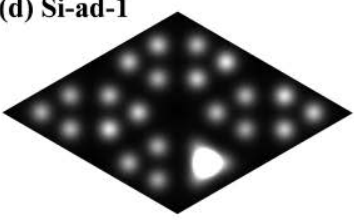

(e) Ag-SV-1

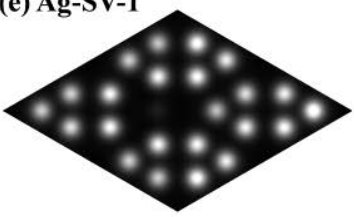

(f) Ag-ad-1

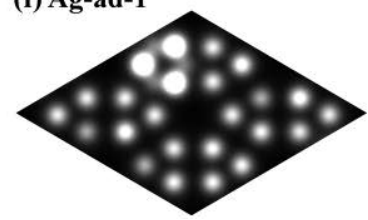

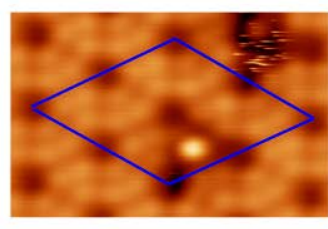
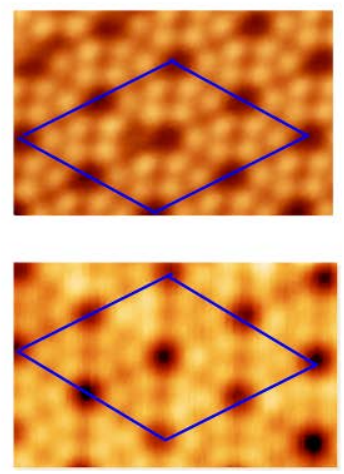

Figure 2. Simulated (left panels) and experimental (right panels) STM images for (a) perfect $4 \times 4$ silicene and for defective $4 \times 4$ silicene with (b) Si-SV-1, (c) Si-DV-1, (d) Si-ad-1, (e) Ag-SV-1, and (f) Ag-ad-1. The blue rhombus indicates the simulation supercell of $4 \times 4$ silicene. The bias voltages for the experimental STM images are $1.2 \mathrm{~V}$ for (a), $1.0 \mathrm{~V}$ for (b), $0.2 \mathrm{~V}$ for (c), $0.2 \mathrm{~V}$ for (d), $-2.0 \mathrm{~V}$ for (e), and $0.8 \mathrm{~V}$ for (f), respectively.

Various structural defects in silicene sheets have been observed in previous experiments [34-35, 51-57]. The atomic structures of these defects are still unclear, however, due to the buckling structure of epitaxial silicene on metal substrates. To recognize the true defect structures in epitaxial growth of silicene and thus end the confusion, the STM images of all the defective silicene/Ag(111) superstructures constructed in this work were simulated and compared with the STM images from our own experiments. Firstly, the simulated STM image for perfect $4 \times 4$ silicene on $\mathrm{Ag}(111)$ agrees well with that obtained experimentally (Figure 2a). As shown in Figure 2b, the simulated STM image of Si-SV-1 exhibits a semilunar black hole, which coincides well with the experimental image. Careful observation tells us that three bright points are lost in the STM image of Si-SV-1 compared to the complete silicene lattice. The simulated STM image of Si-DV-1 (Figure 2c) shows a bigger black hole than that in Si-SV-1, since one more Si atom is missing. Si-ad-1, which is the most stable configuration for the Si adatom on $4 \times 4$ silicene, exhibits a large bright point in the STM image, as displayed in Figure 2d. Its apparent height is $1.45 \AA$ higher than that of the buckled-up Si atoms (Figure 1d). The simulated STM image is also in accordance with 
the experimental image, in which one big bright point replaces the original three bright points in a triangle and the other bright points are nearly unaffected.

As discussed above, in $4 \times 4$ silicene with Ag-SV-1, one buckled-up Si atom in the original $4 \times 4$ silicene would become buckled down due to the missing Ag atom underneath. The local density of states will be affected, which results in the absence of an individual bright point in the simulated STM image for Ag-SV-1, as shown in Figure 2e. For Ag-ad-1, three original bright points arranged in a triangle are enhanced in the STM images, as shown in Figure 2f. This is because the Ag adatom lifts up the Si atoms above it and thus enhances the local density of states (LDOS). The excellent agreement between the simulated and experimental STM images for Ag-SV-1 and Ag-ad-1 confirms the presence of the structural defects in the Ag(111) surface underneath, in addition to those in the silicene sheet. This may also explain why it is hard to identify the point defects in epitaxial silicene grown on $\mathrm{Ag}(111)$ solely from the STM measurements in previous experiments.

\subsection{Structures of various defects in $\sqrt{13} \times \sqrt{13}$ silicene}

For $\sqrt{13} \times \sqrt{13}$ silicene, there are two phases, namely $\sqrt{13} \times \sqrt{13}$-I and $\sqrt{13} \times \sqrt{13}$-II [53]. Here, we only focus on $\sqrt{13} \times \sqrt{13}$-I since it is well studied and its atomic structure is well known. Usually, $\sqrt{13} \times \sqrt{13}$-II phase does not possess long range order and shows a large hexagonal pattern [55]; thus it is not considered here. Thereafter, here $\sqrt{13} \times \sqrt{13}$ silicene means $\sqrt{13} \times \sqrt{13}$-I silicene if there is no extra illustration. The atomic structures and simulated STM images of perfect $\sqrt{13} \times \sqrt{13}$ silicene are shown in Figure 3a. In our model of $\sqrt{13} \times \sqrt{13}$ silicene, there are four buckled-up Si atoms in one supercell, corresponding to four bright points in the STM image from both the DFT simulation and our experiment. Generally speaking, the Si-SW, Si-SV, and Si-DV defects in a $\sqrt{13} \times \sqrt{13}$ silicene sheet share similar characteristics to those in a $4 \times 4$ silicene sheet, as displayed in Figure 3, and Figure S4 and Figure S5 in the Supporting Information. Si-SV-1 (Figure 3b), in which one buckled-up silicon atom is missing, is the most stable configuration for Si-SV defects in $\sqrt{13} \times \sqrt{13}$ silicene, with ultralow formation energy of only $0.052 \mathrm{eV}$. On the other hand, the formation energies for Si-SV-2, Si-SV-3, and Si-SV-4 defects (Figure S5), in which the vacancy site corresponds to the buckled-down silicon atom, are much higher (0.148-0.534 eV). 
Similar to the case of the single vacancy, for a double vacancy with one missing buckled-up atom and one missing buckled-down atom, e.g., Si-DV-1 (Figure 3c), the formation energy is as low as $0.08 \mathrm{eV}$. Since there is only one lost buckled-up silicon atom at most, Si-SV and Si-DV defects in $\sqrt{13} \times \sqrt{13}$ silicene exhibit the same STM image, which is a perfect STM image with the lack of one bright point, as shown in Figure 3b and c. The excellent agreement between the simulated and experimental STM images for Si-SV and Si-DV clarifies that the missing bright point observed experimentally corresponds to the silicon vacancy defect. Note that the most preferred adsorption site for a $\mathrm{Si}$ adatom on $\sqrt{13} \times \sqrt{13}$ silicene is no longer the hollow site, but the top site, as displayed in Figure S4e and $4 \mathrm{f}$ of the Supporting Information.

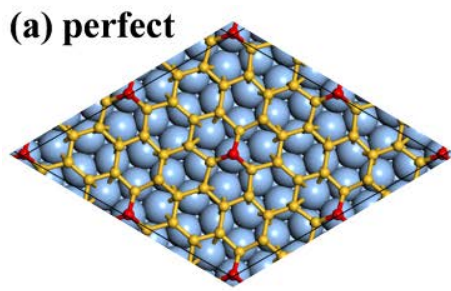

(b) Si-SV-1
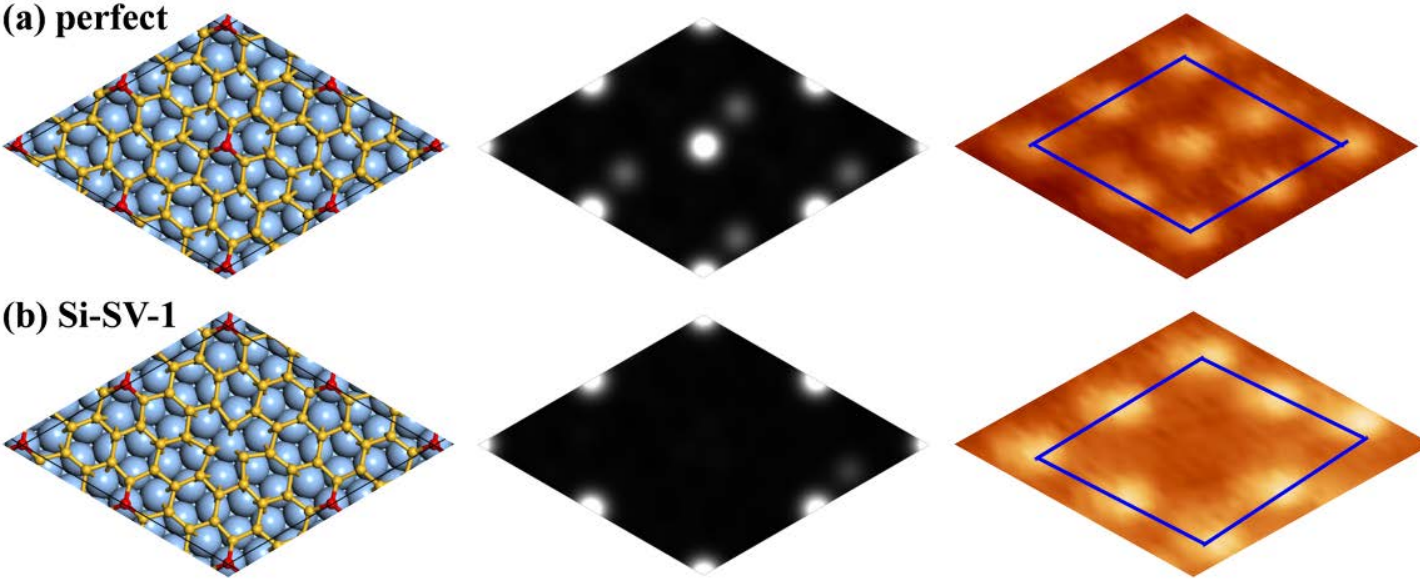

(c) $\mathrm{Si}-\mathrm{DV}-1$
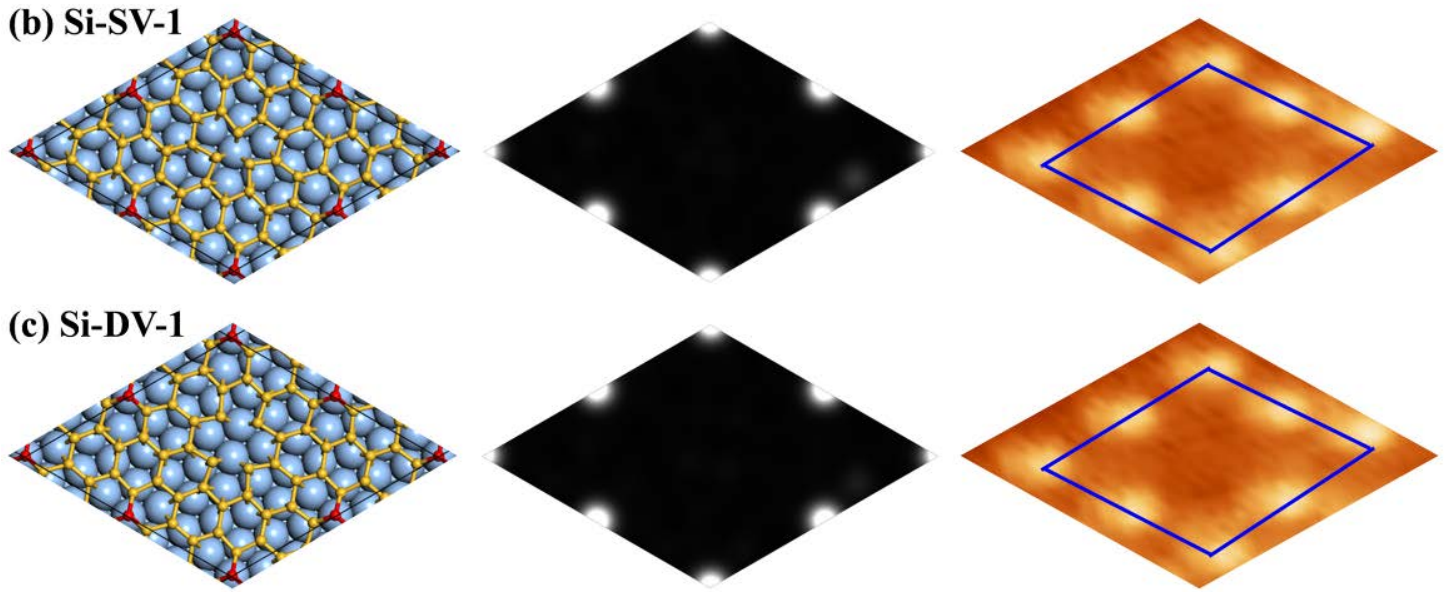

Figure 3. Atomic structures (left panels), and simulated (middle panels) and experimental (right panels) STM images of (a) perfect $\sqrt{13} \times \sqrt{13}$ silicene, (b) $\sqrt{13} \times \sqrt{13}$ silicene with silicon SV (Si-SV-1), and (c) $\sqrt{13} \times \sqrt{13}$ silicene with silicon DV (Si-DV-1). The sky-blue, yellow, and red balls represent Ag atoms, buckled-down Si atoms, and buckled-up Si atoms, respectively. The black rhombus in the atomic structures indicates the simulation supercell, which is also represented by the blue rhombus in the experimental STM images. The bias voltages for the experimental STM images are $-0.5 \mathrm{~V}$, and the tip current, $I_{\text {tip }}=4 \mathrm{nA}$.

2.3 Structures of various defects in $2 \sqrt{3} \times 2 \sqrt{3}$ silicene 
The atomic structures and simulated STM images of perfect $2 \sqrt{3} \times 2 \sqrt{3}$ silicene are shown in Figure 4a. The simulated STM image of $2 \sqrt{3} \times 2 \sqrt{3}$ silicene shows bright points arranged in a honeycomb lattice, in accordance with experimental STM displayed in Figure 4c. However, in experiments, $2 \sqrt{3} \times 2 \sqrt{3}$ silicene always appear disordered (Figure 4c). Atomic structures and simulated STM of all considered defective $2 \sqrt{3} \times 2 \sqrt{3}$ silicene can be found in Figure S6, S7, S8 and S9 in the Supporting Information. Overall speaking, the structures of Si-SW, Si-SV, Si-DV and Si-ad defects in $2 \sqrt{3} \times 2 \sqrt{3}$ silicene are similar to those in $4 \times 4$ and $\sqrt{13} \times \sqrt{13}$ silicene. While for Si-SV in $2 \sqrt{3} \times 2 \sqrt{3}$ silicene, the reconstructed configuration proposed previously for freestanding silicene [40] is more energetically favorable than the configuration with three two-coordination silicon atoms. This is different from those in $4 \times 4$ and $\sqrt{13} \times \sqrt{13}$ silicene. For Si-ad in $2 \sqrt{3} \times 2 \sqrt{3}$ silicene, the most stable adsorption site is the top site, same as that in $\sqrt{13}$ $\times \sqrt{13}$ silicene, but different from that in $4 \times 4$ silicene.

In sharp contrast with $4 \times 4$ and $\sqrt{13} \times \sqrt{13}$ silicene, most of the defects in $2 \sqrt{3} \times 2 \sqrt{3}$ silicene, including Si-SW, Si-SV, Si-DV and Si-ad, do not lead to absence of bright points in the STM images but induce disorder of bright points instead (Figure 4b and Figure S6, S7, S8 and S9). Specially, Si-DV-1 in $2 \sqrt{3} \times 2 \sqrt{3}$ silicene would result in a small displacement of one bright point in the STM image of perfect silicene. Therefore, a warped hexagon occurs in the STM image (denoted by a green circle in Figure 4b), which can be frequently seen in the experimental STM image (denoted by green circles in Figure 4c). 
(a) perfect

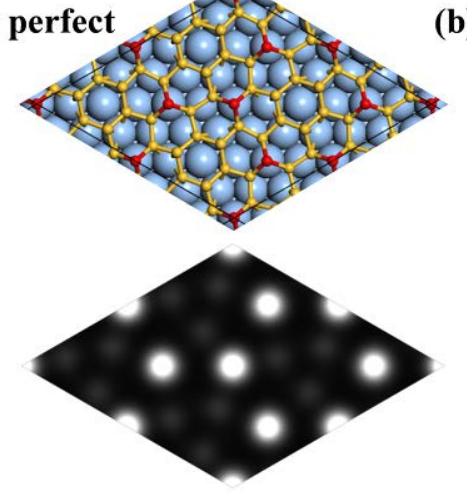

(b) Si-DV-1

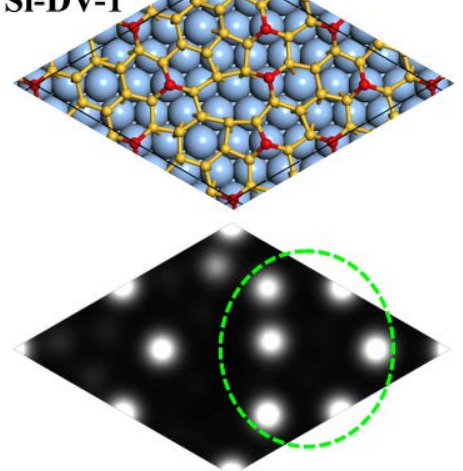

(c) experimental STM

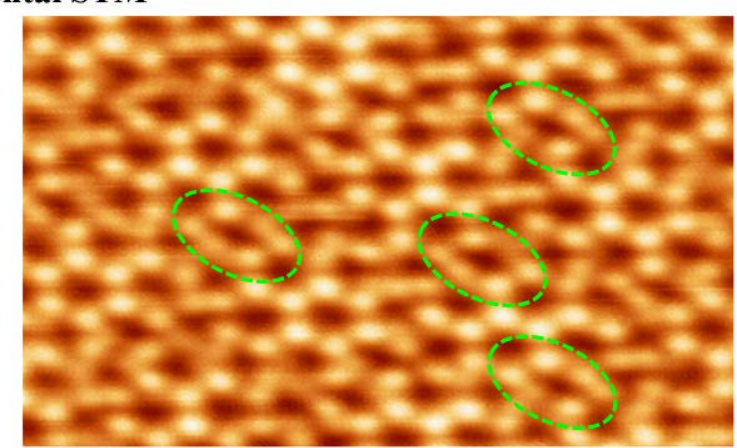

Figure 4. (a) Atomic structures and simulated STM images of perfect $2 \sqrt{3} \times 2 \sqrt{3}$ silicene. (b) Atomic structures and simulated STM images of $2 \sqrt{3} \times 2 \sqrt{3}$ silicene with silicon DV (Si-DV-1). (c) Experimental STM images of $2 \sqrt{3} \times 2 \sqrt{3}$ silicene. The sky-blue, yellow, and red balls in (a) and (b) represent Ag atoms, buckled-down Si atoms, and buckled-up Si atoms, respectively. The black rhombus in the atomic structures indicates the simulation supercell. The bias voltages for the experimental STM images are $-0.8 \mathrm{~V}$ and the tip current, $I_{\text {tip }}=4 \mathrm{nA}$.

\subsection{Diffusion of defects}

As the growth temperature of silicene is typically $480-550 \mathrm{~K}$ [31-32, 34, 36], point defects may diffuse or aggregate, which would affect the distribution of defects and final quality of the epitaxial silicene sheet. Considering this, we further explore the possible diffusion behavior of Si-SV and Si-ad in $4 \times 4, \sqrt{13} \times \sqrt{13}$ and $2 \sqrt{3} \times 2 \sqrt{3}$ silicene using the climbing-image nudged elastic band (cNEB) method [64]. A schematic plot for the diffusion of a Si-SV in $4 \times 4$ silicene is shown in Figure 5. The initial configuration is set to be Si-SV-1, which is the most stable for an Si-SV, while the final configuration is Si-SV-2. The transition state shown in Figure 5 possesses the same configuration as Si-SV-1 but different position for the missing Si atom. The energy barrier for this diffusion is only $0.47 \mathrm{eV}$. Actually, when a Si-SV diffuses from Si-SV-1 to Si-SV-2, 
it can then diffuse from the Si-SV-2 to another Si-SV-1. In other words, a Si-SV can diffuse throughout the whole $4 \times 4$ silicene sheet with a maximal energy barrier of $0.47 \mathrm{eV}$. Also note that the diffusion barrier for an Si-SV in freestanding silicene is only $0.12 \mathrm{eV} \mathrm{[40],} \mathrm{which} \mathrm{is} \mathrm{much}$ lower than the present value. This is because that in the epitaxial silicene, the diffusion of one Si-SV involves breaking of both Si-Si bonds and Si-Ag bonds. While in free-standing silicene, the diffusion of one Si-SV involves breaking of Si-Si bonds only. Thus, the moderate Si-Ag interaction makes the diffusion of Si-SV more difficult.

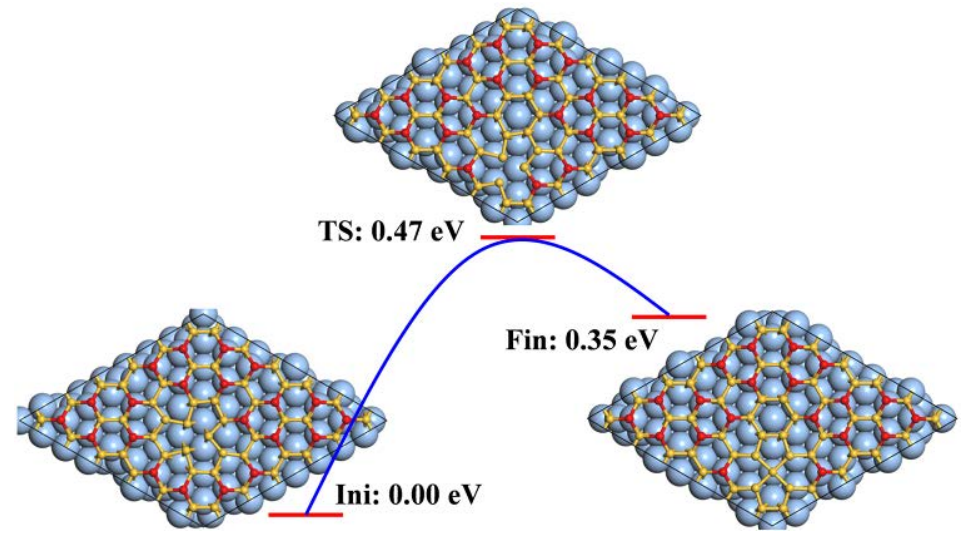

Figure 5. Schematic plots for diffusion of a Si-SV defect in $4 \times 4$ silicene on $\mathrm{Ag}(111)$. The sky-blue, yellow, and red balls represent Ag atoms, buckled-down Si atoms, and buckled-up Si atoms, respectively. The black rhombus indicates the simulation supercell.

The diffusion of a Si adatom in $4 \times 4$ silicene is plotted in Figure 6 , in which the Si adatom migrates from a hollow site (Si-ad-1) to a top site (Si-ad-2) by overcoming an energy barrier of $0.41 \mathrm{eV}$ and then diffuses to another top site, surmounting a barrier of $0.42 \mathrm{eV}$. Two transition states, TS1 and TS2, occur in the process shown in Figure 6. For TS1, the Si adatom deviates from the middle of the hexagon, bonded with two buckled-up Si atoms. For TS2, The Si adatom sits nearly at bridge site of the Si-Si bond. Therefore, a Si adatom can diffuse from one of the most stable sites (hollow site) to another one by overcoming a maximal barrier of $0.42 \mathrm{eV}$, which is much lower than that in freestanding silicene $(1.03 \mathrm{eV})[40]$.

To see how fast Si-SV and Si-ad can diffuse, we estimated the jump frequency, $p$, by [65]:

$$
p \approx v^{\bullet} \exp \left(-E_{a} / k_{B} T\right)
$$

where $v$ is a characteristic atomic vibrational frequency, $E_{a}$ is the activation energy for diffusion, 
$k_{B}$ is the Boltzmann constant, and $T$ is the temperature. The typical magnitude of the atomic vibration frequencies is about $10^{13} \mathrm{~Hz}$. At $500 \mathrm{~K}$, the jump frequency for Si-SV and Si-ad can be as high as $2.0 \times 10^{8} \mathrm{~Hz}$ and $6.2 \times 10^{8} \mathrm{~Hz}$, respectively. As a consequence, Si-SV and Si-ad can diffuse very fast in epitaxial $4 \times 4$ silicene sheet at the growth temperature.

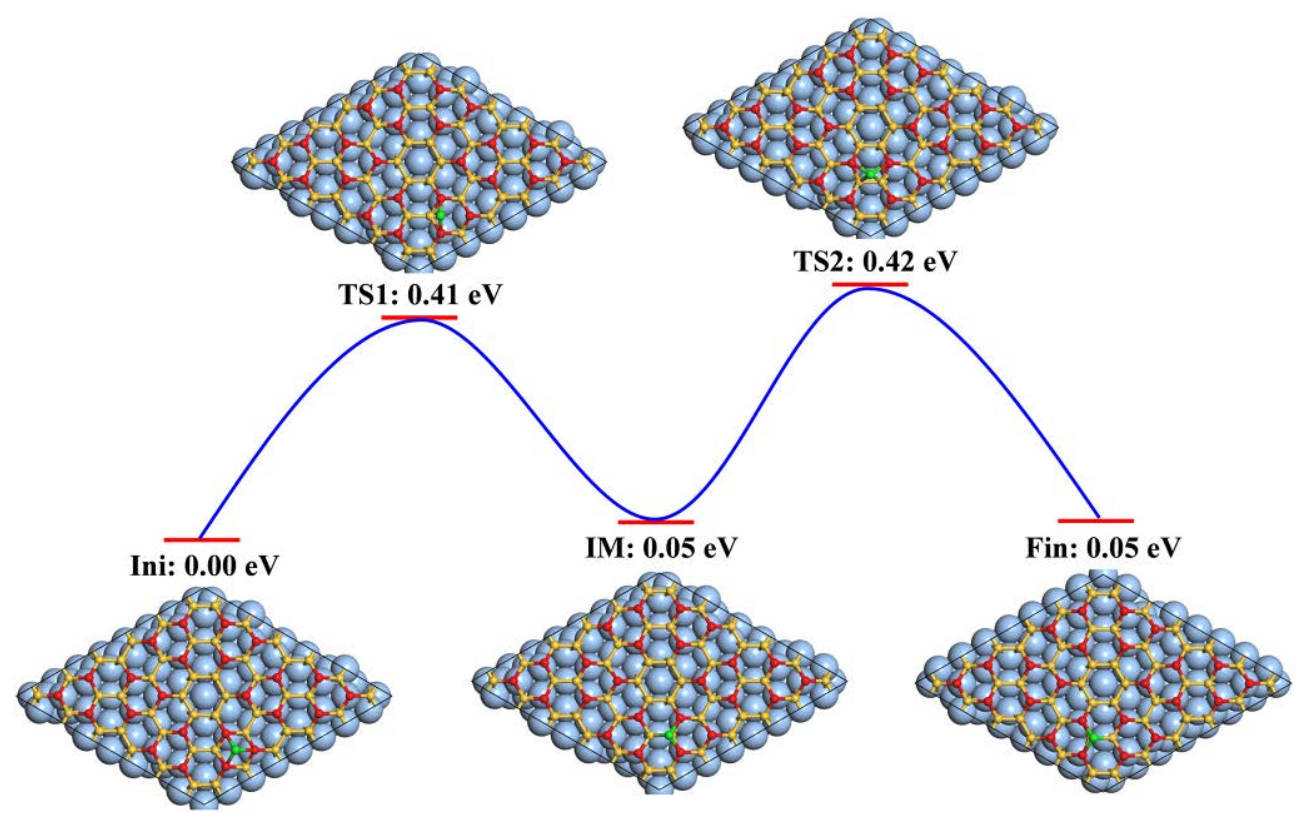

Figure 6. Schematic plots for diffusion of a Si-ad defect in $4 \times 4$ silicene on $\mathrm{Ag}(111)$. The sky-blue, yellow, red, and green balls represent Ag atoms, buckled down Si atoms, buckled up Si atoms, and Si adatoms respectively. The black rhombus indicates the simulation supercell.

For $\sqrt{13} \times \sqrt{13}$ and $2 \sqrt{3} \times 2 \sqrt{3}$ silicene, the diffusion paths of Si-SV and Si-ad are plotted in Figure S10, S11, S12, S13 in the Supporting Information. The maximal activation energies for diffusion of Si-SV in $\sqrt{13} \times \sqrt{13}$ and $2 \sqrt{3} \times 2 \sqrt{3}$ silicene are $0.76 \mathrm{eV}$ and $0.38 \mathrm{eV}$, respectively, which are comparable with that in $4 \times 4$ silicene $(0.47 \mathrm{eV})$. The corresponding jump frequencies at $500 \mathrm{~K}$ are estimated to be $2.0 \times 10^{5} \mathrm{~Hz}(\sqrt{13} \times \sqrt{13})$ and $1.6 \times 10^{9} \mathrm{~Hz}(2 \sqrt{3} \times 2 \sqrt{3})$, respectively. Therefore, a Si single vacancy can still migrate in $\sqrt{13} \times \sqrt{13}$ silicene at an appreciable rate, but the diffusion is slower than that in $4 \times 4$ silicene and $2 \sqrt{3} \times 2 \sqrt{3}$ silicene. The diffusion barriers of Si-ad in $\sqrt{13} \times \sqrt{13}$ and $2 \sqrt{3} \times 2 \sqrt{3}$ silicene are $1.04 \mathrm{eV}$ and $0.98 \mathrm{eV}$, respectively, and the corresponding jump frequencies at $500 \mathrm{~K}$ are estimated to be $3.8 \times 10^{2} \mathrm{~Hz}$ and $1.5 \times 10^{3} \mathrm{~Hz}$, respectively. Thus, diffusion of a Si adatom would be rather slow in $\sqrt{13}$ $\times \sqrt{13}$ and $2 \sqrt{3} \times 2 \sqrt{3}$ silicene. 
Moreover, $a b$ initio molecular dynamics (AIMD) simulations were performed for Si-SV in $4 \times 4, \sqrt{13} \times \sqrt{13}$ and $2 \sqrt{3} \times 2 \sqrt{3}$ silicene to examine the defect diffusion directly. Within the canonical NVT ensemble, the system temperature was set at $550 \mathrm{~K}$, and the time step was $1 \mathrm{fs}$. The snapshot structures for Si-SV migration in $4 \times 4$ silicene are shown in Figure 7 a. After 1.38 ps, the Si-SV moves to one side, with a silicon atom diffusing in the direction indicated by the red arrow. Then, this vacancy moves to a new position in the way indicated by red arrow at $2.12 \mathrm{ps}$. The Si-SV-2 configuration, which is the final state of the diffusion path in Figure 5, does not appear during the entire simulation time of 8 ps. This is because the Si-SV-2 configuration would occur at about $0.2 \mathrm{~ns}$ according to our estimated jump frequency of $5.2 \times 10^{8} \mathrm{~Hz}$ at $550 \mathrm{~K}$, far beyond the time scale of the AIMD simulation. Nevertheless, we can still see the fast diffusion of Si-SV in the silicene sheet. For $\sqrt{13} \times \sqrt{13}$ silicene, the diffusion of a Si-SV is more evident, as shown in Figure 7b. At $2.71 \mathrm{ps,} \mathrm{the} \mathrm{Si-SV} \mathrm{has} \mathrm{already} \mathrm{migrated} \mathrm{far} \mathrm{away} \mathrm{from} \mathrm{its} \mathrm{original} \mathrm{position.} \mathrm{Then,}$ the configuration of Si-SV at 2.71 ps is just the same as for Si-SV-3, which is shown in Figure S5b of the Supporting Information. We can also see very fast diffusion behavior of Si-SV in $2 \sqrt{3} \times$ $2 \sqrt{3}$ silicene as shown in Figure $7 c$, which is expected by the large jump frequency estimated by Eq. (2).

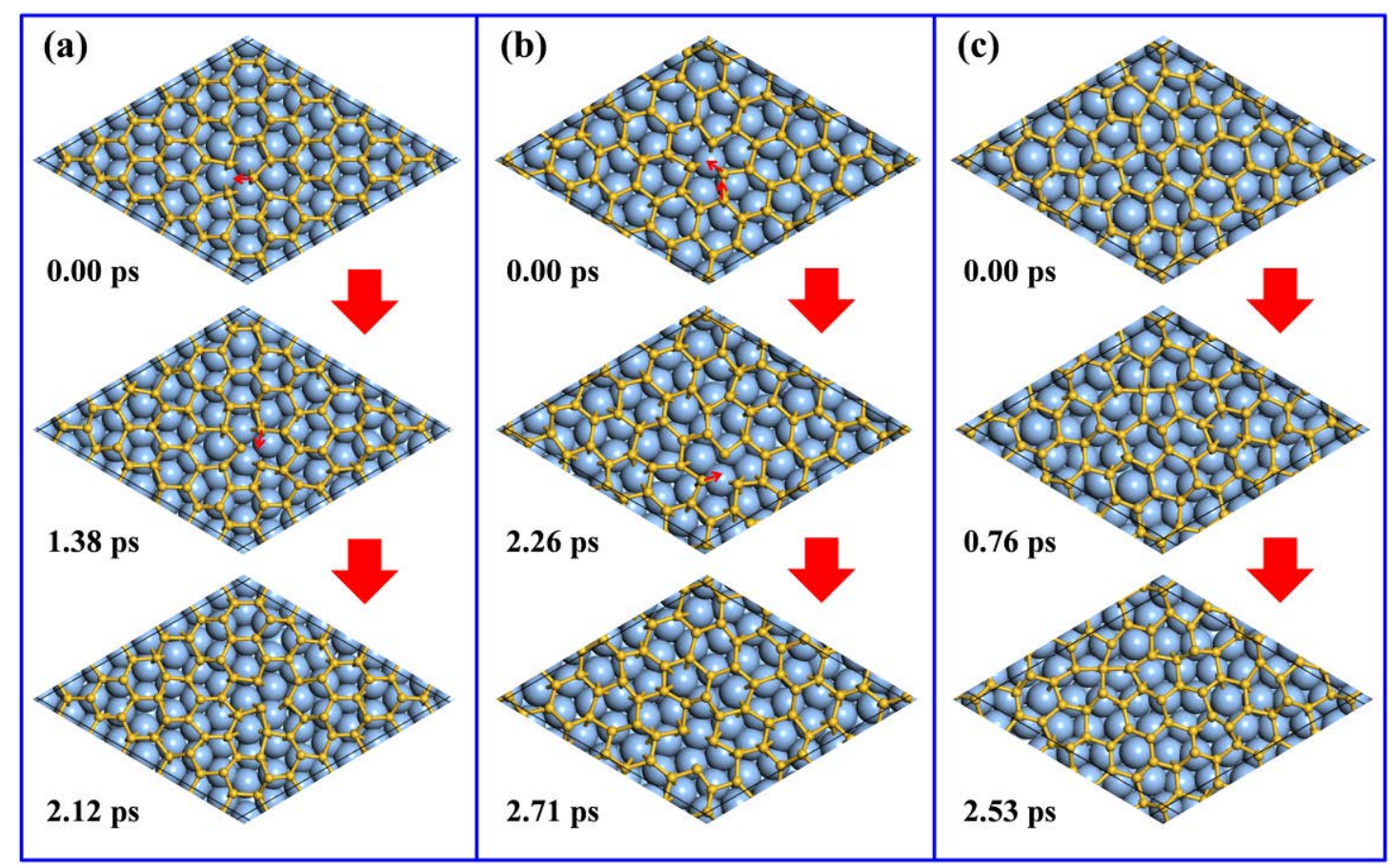

Figure 7. Snapshots from the AIMD simulation of silicene monolayers on $\mathrm{Ag}(111)$ at $550 \mathrm{~K}$ : (a) $4 \times 4$ silicene; (b) $\sqrt{13} \times \sqrt{13}$ silicene; (c) $2 \sqrt{3} \times 2 \sqrt{3}$ silicene. 


\subsection{Formation energies and concentrations of defects}

The concentration of defects in a material directly determines its fundamental properties and is thus a critical concern for device applications. The probability that a point defect occurs at a given site is proportional to the Boltzmann factor for thermal equilibrium: $P=\exp \left(-E_{f o r m} / k_{B} T\right)$, where $E_{\text {form }}$ is the formation energy of the point defect, $k_{B}$ is the Boltzmann constant, $T$ is the temperature [65]. As discussed above, for a given kind of point defect, its formation energy is position dependent. Therefore, for a given kind of point defect, the total number of defects in one unit cell is the sum of the probability $P$ for this kind of point defect occurring at all possible sites. Thus, the concentration $c$ for a given type of defect can be estimated as

$$
c=\sum_{i} \exp \left(-\frac{E_{\text {form }}^{i}}{k_{B} T}\right) / S
$$

where $E_{\text {form }}^{i}$ is the formation energy of one kind of defect at site $i$ in one unit cell, and $S$ is the area of one unit cell. The formation energies for various point defects in the three silicene superstructures are summarized in Table 1. The formation energies for Si-SW defects in $\sqrt{13}$ $\times \sqrt{13}(0.815-1.264 \mathrm{eV})$ and $2 \sqrt{3} \times 2 \sqrt{3}(0.863-1.192 \mathrm{eV})$ silicene are lower than those in $4 \times 4$ silicene (1.354 - $1.544 \mathrm{eV})$, indicating higher concentrations of Si-SW defects in $\sqrt{13} \times \sqrt{13}$ and $2 \sqrt{3} \times 2 \sqrt{3}$ silicene. It is noteworthy that the formation energies for Si-SV and Si-DV defects in $\sqrt{13} \times \sqrt{13}$ and $2 \sqrt{3} \times 2 \sqrt{3}$ silicene are extremely low, suggesting that epitaxial $\sqrt{13} \times \sqrt{13}$ and $2 \sqrt{3} \times 2 \sqrt{3}$ silicene on $\operatorname{Ag}(111)$ would be very defective (which will be further discussed later). For all the three silicene sheets, the formation energy of a Si-DV is much lower than that of two Si-SVs, indicating that two Si-SV defects would coalesce into one Si-DV defect by overcoming a moderate energy barrier $(0.47 \mathrm{eV}$ for $4 \times 4$ silicene, $0.76 \mathrm{eV}$ for $\sqrt{13} \times \sqrt{13}$ silicene

and $0.38 \mathrm{eV}$ for $2 \sqrt{3} \times 2 \sqrt{3}$ silicene). In the experimental STM image of $\sqrt{13} \times \sqrt{13}$ silicene (Figure 8), there are abundant big black holes and lines of lost bright points, which might originate from coalescing of Si-SV or Si-DV defects. Moreover, the distribution of bright points in the STM image is inhomogeneous due to the easy merging of Si-SVs via diffusion. 
Table 1. Formation energies (in unit of eV) for various point defects in $4 \times 4, \sqrt{13} \times \sqrt{13}$ and $2 \sqrt{3} \times 2 \sqrt{3}$ silicene superstructures.

\begin{tabular}{|c|c|c|c|c|}
\hline & Si-SW & Si-SV & Si-DV & Si-ad \\
\hline $4 \times 4$ & $1.354-1.544$ & $0.731-1.059$ & $0.890-1.163$ & $0.705-0.894$ \\
\hline$\sqrt{13} \times \sqrt{13}$ & $0.815-1.264$ & $0.052-0.534$ & $0.079-0.839$ & $0.721-1.210$ \\
\hline $2 \sqrt{3} \times 2 \sqrt{3}$ & $0.863-1.192$ & $0.213-0.655$ & $0.098-0.358$ & $0.249-1.052$ \\
\hline
\end{tabular}

The equilibrium concentrations of various defects in the three silicene superstructures are estimated by Eq. (3) and listed in Table 2. According to Eq. (3), since the concentration depends on the growth temperature of silicene on the $\mathrm{Ag}(111)$ surface, which is around $500 \mathrm{~K}$ [31-32, 34, 36], the temperature in Eq .(3) is set to be $500 \mathrm{~K}$. We first discuss the equilibrium concentration of defects in $4 \times 4$ silicene. The equilibrium concentration of Si-SW defect is as small as $14 \mathrm{~cm}^{-2}$, which means that there is only one Si-SW defect in a large area of $7 \mathrm{~mm}^{2}$ on average. Thus, the Si-SW defect can hardly be observed in experiment. Compared to Si-SW, the equilibrium concentrations of Si-SV, Si-DV, and Si-ad are larger. The estimated concentration of Si-SV is $4.9 \times$ $10^{7} \mathrm{~cm}^{-2}$, which is much larger than that of Si-DV $\left(4.6 \times 10^{5} \mathrm{~cm}^{-2}\right)$. Si-SV diffuses very fast during the growth process, however, and two Si-SVs can coalesce into one Si-DV to lower the energy. As a result, the final equilibrium concentration of Si-DV should be larger than $4.6 \times 10^{5} \mathrm{~cm}^{-2}$, and the concentration of Si-SV should be smaller than $4.9 \times 10^{7} \mathrm{~cm}^{-2}$. Nevertheless, Si-SV and Si-DV in $4 \times 4$ silicene have relatively small equilibrium concentrations but should still be observed occasionally. The estimated concentration of Si-ad is $2.9 \times 10^{7} \mathrm{~cm}^{-2}$, which is comparable to that of Si-SV. Actually, in our experiments we do observe Si-SV, Si-DV, and Si-ad defects, but not Si-SW defects. 
Table 2. Equilibrium concentrations at $500 \mathrm{~K}$ for various point defects in $4 \times 4, \sqrt{13} \times \sqrt{13}$ and $2 \sqrt{3} \times 2 \sqrt{3}$ silicene superstructures in unit of $\mathrm{cm}^{-2}$.

\begin{tabular}{|c|c|c|c|c|}
\hline & Si-SW & Si-SV & Si-DV & Si-ad \\
\hline $4 \times 4$ & 14 & $4.9 \times 10^{7}$ & $4.6 \times 10^{5}$ & $2.9 \times 10^{7}$ \\
\hline$\sqrt{13} \times \sqrt{13}$ & $3.1 \times 10^{6}$ & $4.4 \times 10^{13}$ & $5.0 \times 10^{13}$ & $2.5 \times 10^{7}$ \\
\hline $2 \sqrt{3} \times 2 \sqrt{3}$ & $1.0 \times 10^{6}$ & $4.9 \times 10^{12}$ & $1.2 \times 10^{14}$ & $1.3 \times 10^{12}$ \\
\hline
\end{tabular}

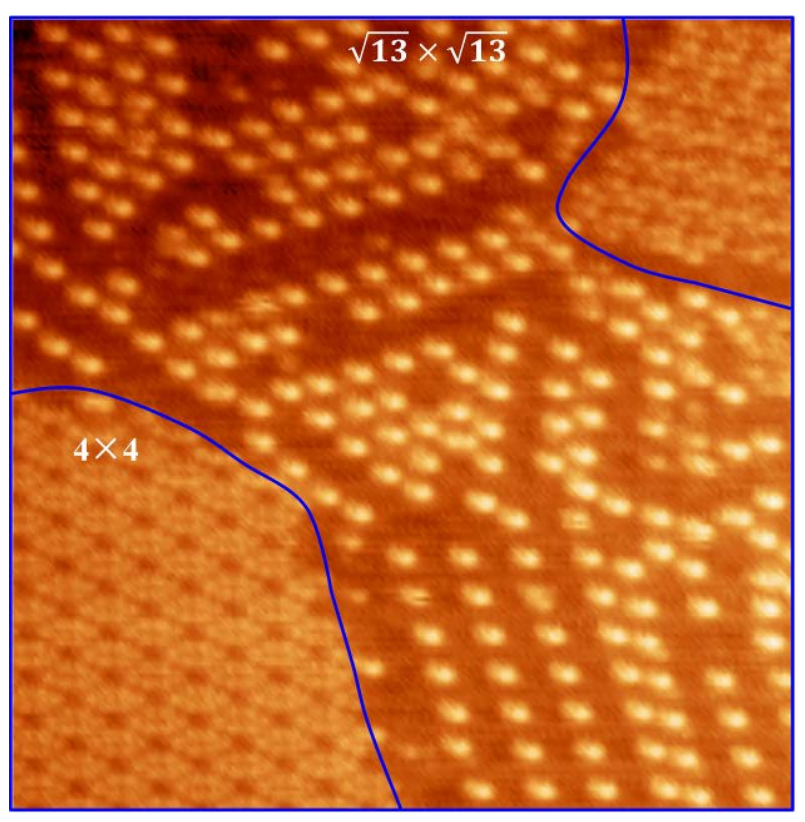

Figure 8. Large scale STM image showing $4 \times 4$ and $\sqrt{13} \times \sqrt{13}$ silicene from experiment $\left(V_{\text {bias }}=\right.$ $\left.-1.5 \mathrm{~V}, I_{\text {tip }}=4 \mathrm{nA}\right)$.

Now, let's turn to $\sqrt{13} \times \sqrt{13}$ silicene. As shown in Table 2, the equilibrium concentration of Si-SW defects in $\sqrt{13} \times \sqrt{13}$ silicene $\left(3.1 \times 10^{6} \mathrm{~cm}^{-2}\right)$ is much larger than that in $4 \times 4$ silicene. It is still hard to find a Si-SW defect, since only one Si-SW defect exists in an area of $33.3 \mu \mathrm{m}^{2}$. Note that Si-SV and Si-DV have very high concentrations of about $5.0 \times 10^{13} \mathrm{~cm}^{-2}$ in $\sqrt{13} \times \sqrt{13}$ silicene, which means that there should be one Si-SV or Si-DV in every $2 \mathrm{~nm}^{2}$. The large concentration of vacancy defects is clearly reflected in the experimental STM image in Figure 8. Combining the STM observations with the above theoretical results, we conjecture that the observed big black holes and lines of black holes correspond to vacancy clusters from coalescence of Si-SV and Si-DV defects. Consequently, the total concentration of vacancy defects can be 
obtained by counting the number of missing bright points in the STM image. In such a way, the concentration of missing bright points is $4.1 \times 10^{13} \mathrm{~cm}^{-2}$ for $\sqrt{13} \times \sqrt{13}$ silicene in Figure 8, which is close to the equilibrium concentration of Si-SV or Si-DV calculated from Eq. (3) in Table 2. The accordance between the calculation and experiment demonstrates that our theoretical method is reliable for describing the defect concentration qualitatively. The Si-ad in $\sqrt{13} \times \sqrt{13}$ silicene has a concentration of $2.5 \times 10^{7} \mathrm{~cm}^{-2}$, which is nearly the same as that in $4 \times 4$ silicene. Nevertheless, we have not observed Si-ad in $\sqrt{13} \times \sqrt{13}$ silicene in our experiments as yet.

Finally, we discuss the mysterious $2 \sqrt{3} \times 2 \sqrt{3}$ silicene based on our results, though there is now some uncertainty and debate on this phase [66-67]. The concentration of Si-SW defects in $2 \sqrt{3} \times 2 \sqrt{3}$ and $\sqrt{13} \times \sqrt{13}$ silicene are in the same order of magnitude, thus it is also hard to find Si-SW defects in $2 \sqrt{3} \times 2 \sqrt{3}$ silicene. It is noteworthy that the concentrations of Si-SV and Si-DV in $2 \sqrt{3} \times 2 \sqrt{3}$ silicene are very large, which are comparable to that in $\sqrt{13} \times \sqrt{13}$ silicene. However, Si-SV and Si-DV would not induce absence but disorder of bright points in the STM images as discussed above. Especially, Si-DV-1 shown in Figure 4b would result in bright points arranged in a warped hexagon. Hence we did not see prominent absence of bright points in the experimental STM image, but we did observe large number of warped hexagon (Figure 4c), which may originate from the large concentration of Si-DV. Recently, a geometry model of $2 \sqrt{3} \times$ $2 \sqrt{3}$ silicene was proposed, in which silicene layer is based on periodic arrangements of perfect areas of $2 \sqrt{3} \times 2 \sqrt{3}$ silicene surrounded by defect areas on a rigid lattice of silver [67]. In that model, the warped hexagons observed in experimental STM image are ascribed to local relaxation of strain during epitaxial growth rather than point defects. Our results, however, give another possible explanation for the disordered appearance of $2 \sqrt{3} \times 2 \sqrt{3}$ silicene in experiments. Finally, in $2 \sqrt{3} \times 2 \sqrt{3}$ silicene, the Si-ad also has a large concentration comparable to that of Si-SV. Though Si-ad diffuses slowly in $2 \sqrt{3} \times 2 \sqrt{3}$ silicene, Si-SV can diffuse really fast. When a Si-SV meets a Si-ad, they may recombine and thus heal each other. Nevertheless, the final concentration of Si-DV should be very large in $2 \sqrt{3} \times 2 \sqrt{3}$ silicene due to its very small formation energy and easy coalescence of Si-SVs.

Based on the discussion above, we can see that the equilibrium concentrations of point defects are generally very small in $4 \times 4$ silicene, but could be rather large in $\sqrt{13} \times \sqrt{13}$ and $2 \sqrt{3} \times 2 \sqrt{3}$ phases. This nicely explains our experimental observation that $4 \times 4$ silicene appears perfect, 
while $\sqrt{13} \times \sqrt{13}$ and $2 \sqrt{3} \times 2 \sqrt{3}$ silicene looks defective (Figure 8 and Figure 4c). In addition, in $4 \times 4$ and $2 \sqrt{3} \times 2 \sqrt{3}$ silicene, Si-ad has a concentration comparable to that of Si-SV, and either Si-ad or Si-SV can diffuse very fast throughout the entire silicene sheet with a diffusion barrier of about $0.4 \mathrm{eV}$. When a Si adatom diffuses to a Si-SV site or vice versa, the Si-SV can capture the Si adatom and the defect is thus healed. Therefore, appropriate annealing may help improve the quality of epitaxial $4 \times 4$ and $2 \sqrt{3} \times 2 \sqrt{3}$ silicene. Generally, $4 \times 4$ silicene should be fabricated for future nanoelectronic devices due to its high quality and easy self-healing capability with respect to defects.

\section{Conclusion}

To summarize, the morphologies and energetics of various point defects in epitaxial silicene on $\operatorname{Ag}(111)$ surfaces have been systematically investigated using atomistic first-principles calculations combined with experimental scanning tunneling microscopy. The atomic structures for these point defects observed in experimental STM are identified with the aid of DFT calculations. Both cNEB calculations and AIMD simulations demonstrate that Si-SV can diffuse very fast in all silicene superstructures at $500 \mathrm{~K}$. Thus, two Si-SVs would coalesce into one Si-DV via diffusion to lower energy. Moreover, appropriate annealing can help improve the quality of epitaxial silicene since either Si-ad or Si-SV can diffuse very fast in silicene at $500 \mathrm{~K}$ and thus self-healing of Si-ad and Si-SV defects may occur. At $500 \mathrm{~K}$, the equilibrium concentration of Si-SW defects in $4 \times 4$ silicene is as low as $14 \mathrm{~cm}^{-2}$. The concentrations of Si-SV, Si-DV, and Si-ad defects are also very small, i.e. $4.9 \times 10^{7} \mathrm{~cm}^{-2}, 4.6 \times 10^{5} \mathrm{~cm}^{-2}$, and $2.9 \times 10^{7} \mathrm{~cm}^{-2}$, respectively. On the contrary, the estimated concentrations of Si-SV and Si-DV defects in $\sqrt{13} \times \sqrt{13}$ and $2 \sqrt{3} \times$ $2 \sqrt{3}$ silicene are as high as $10^{13} \sim 10^{14} \mathrm{~cm}^{-2}$. The large concentration of point defects and easy diffusion and coalescence of Si-SV nicely explain the defective appearance of $\sqrt{13} \times \sqrt{13}$ and $2 \sqrt{3} \times 2 \sqrt{3}$ silicene in experiments. Therefore, epitaxial $4 \times 4$ silicene is thought to be most suitable monolayer silicene phase for future device applications due to the small amount of defects.

\section{Methods}

All first-principles calculations were carried out using the Vienna Ab Initio Simulation Package 
(VASP) based on DFT [68]. The electron-ion interactions were described by the projector augmented wave (PAW) potentials [69]. The Perdew-Burke-Ernzerhof (PBE) functional within the generalized gradient approximation (GGA) [70] was adopted. A kinetic energy cutoff of $400 \mathrm{eV}$ for the plane wave basis and a convergence criterion of $10^{-4} \mathrm{eV}$ for the total energies were adopted.

The Ag(111) surface was modeled by a three-layer slab model with a vacuum space of more than $12 \AA$, which was cleaved from bulk face-centered-cubic (fcc) silver with the experimental lattice constant of $2.89 \AA$ A. With fixed supercell parameters, the three-layer slab model was further relaxed, with the bottom layer fixed to mimic a semi-infinite solid. Here, we built three common silicene superstructures on the $\operatorname{Ag}(111)$ surface, i.e. $3 \times 3$ silicene on a $4 \times 4 \operatorname{Ag}(111)$ surface, $\sqrt{7}$ $\times \sqrt{7}$ silicene on $\sqrt{13} \times \sqrt{13} \operatorname{Ag}(111)$ surface and $\sqrt{7} \times \sqrt{7}$ silicene on $2 \sqrt{3} \times 2 \sqrt{3} \operatorname{Ag}(111)$, by compressing or stretching the silicene lattice slightly to fit the metal surface, following our previous work [58]. To simulate defective silicene supported on Ag substrate, one point defect was created in a $2 \times 2$ supercell of a silicene on $\mathrm{Ag}(111)$ superstructure to avoid the interactions between adjacent periodic images of the defects in the lateral directions. The lattice constants of our simulation supercells were $23.120 \AA, 20.834 \AA$ and $20.019 \AA$ for $4 \times 4, \sqrt{13} \times \sqrt{13}$ and $2 \sqrt{3} \times$ $2 \sqrt{3}$ silicene, corresponding to $\mathrm{Si}_{72} \mathrm{Ag}_{192}, \mathrm{Si}_{56} \mathrm{Ag}_{156}$ and $\mathrm{Si}_{56} \mathrm{Ag}_{144}$, respectively. The STM images were simulated by using the Tersoff-Hamann approximation [71] with a constant height of $2 \AA$ above the buckled-up Si atoms. Different bias voltages were tested and found to have virtually no influence on the simulated STM images. Thus, the bias voltage was set to be $-1.5 \mathrm{eV}$ for all STM simulations in this paper.

All samples used in this work were fabricated in a preparation chamber supplied with a low-temperature STM/scanning near-field optical microscopy system (LT-STM-SNOM, SNOM1400, Unisoku Co.), as reported elsewhere [29, 72-73]. Clean Ag(111) substrates were prepared by argon ion sputtering and annealed at $800 \mathrm{~K}$ for several cycles. The silicene monolayers were then grown on the $\operatorname{Ag}(111)$ surfaces by evaporation of silicon from a heated silicon wafer. All the measurements were carried out in ultrahigh vacuum (UHV) at $77 \mathrm{~K}$. Pt/Ir tips were calibrated on a silver surface before STM measurements. 
This work was supported by the National Natural Science Foundation of China (11134005, 11375228, 11574040, 11575227), Project 1G2009312311750101 of the Chinese Academy of Sciences, the Australian Research Council (ARC) through a Discovery Project (DP140102581), the University of Wollongong through a University Research Council (URC) Small Grant in 2014, and ARC Linkage Infrastructure, Equipment and Facilities (LIEF) grants (LE100100081 and LE110100099). 


\section{References}

[1] Kara A, Enriquez H, Seitsonen A P, Lew Yan Voon L C, Vizzini S, Aufray B, Oughaddou H 2012 A review on silicene - new candidate for electronics Surf. Sci. Rep. 67 1-18.

[2] Deepthi J, Ayan D 2013 Structures and chemical properties of silicene: Unlike graphene Accounts Chem. Res. 47 593-602.

[3] Liu H, Gao J, Zhao J 2014 Silicene on substrates: Interaction mechanism and growth behavior $J$. Phys.: Conf. Series 491012007.

[4] Yan Voon L C L, Guzmán-Verri G G 2014 Is silicene the next graphene? MRS Bull. 39 366-73.

[5] Dimoulas A 2015 Silicene and germanene: Silicon and germanium in the "flatland" Microelectron. Eng. 131 68-78.

[6] Zhao J et al. 2016 Rise of silicene: A competitive 2D material Prog. Mater. Sci. 83 24-151.

[7] Cahangirov S, Topsakal M, Aktürk E, Şahin H, Ciraci S 2009 Two- and one-dimensional honeycomb structures of silicon and germanium Phys. Rev. Lett. 102236804.

[8] Ding Y, Ni J 2009 Electronic structures of silicon nanoribbons Appl. Phys. Lett. 95083115.

[9] Pan L, Liu H J, Wen Y W, Tan X J, Lv H Y, Shi J, Tang X F 2011 First-principles study of monolayer and bilayer honeycomb structures of group-iv elements and their binary compounds Phys. Lett. A 375 614-9.

[10] Şahin H, Cahangirov S, Topsakal M, Bekaroglu E, Akturk E, Senger R, Ciraci S 2009 Monolayer honeycomb structures of group-iv elements and iii-v binary compounds: First-principles calculations Phys. Rev. B 80155453.

[11] Chen L, Liu C-C, Feng B, He X, Cheng P, Ding Z, Meng S, Yao Y, Wu K 2012 Evidence for dirac fermions in a honeycomb lattice based on silicon Phys. Rev. Lett. 109056804.

[12] Shao Z-G, Ye X-S, Yang L, Wang C-L 2013 First-principles calculation of intrinsic carrier mobility of silicene J. Appl. Phys. 114093712.

[13] Liu C-C, Feng W, Yao Y 2011 Quantum spin hall effect in silicene and two-dimensional germanium Phys. Rev. Lett. 107076802.

[14] Li X, Mullen J, Jin Z, Borysenko K, Buongiorno Nardelli M, Kim K W 2013 Intrinsic electrical transport properties of monolayer silicene and $\mathrm{MoS}_{2}$ from first principles Phys. Rev. B 87115418.

[15] Rojas-Cuervo A M, Fonseca-Romero K M, Rey-González R R 2014 Anisotropic dirac cones in monoatomic hexagonal lattices: A dft study Eur. Phys. J. B 8767.

[16] Novoselov K S, Geim A K, Morozov S V, Jiang D, Zhang Y, Dubonos S V, Grigorieva I V, Firsov A A 2004 Electric field effect in atomically thin carbon films Science 306 666-9.

[17] Berger C et al. 2006 Electronic confinement and coherence in patterned epitaxial graphene Science 312 1191-6.

[18] Novoselov K S, Geim A K, Morozov S V, Jiang D, Katsnelson M I, Grigorieva I V, Dubonos S V, Firsov A A 2005 Two-dimensional gas of massless dirac fermions in graphene Nature 438 197-200.

[19] Knox K R, Wang S, Morgante A, Cvetko D, Locatelli A, Mentes T O, Niño M A, Kim P, Osgood R M 2008 Spectromicroscopy of single and multilayer graphene supported by a weakly interacting substrate Phys. Rev. B 78201408.

[20] Castro Neto A H, Guinea F, Peres N M R, Novoselov K S, Geim A K 2009 The electronic properties of graphene Rep. Prog. Phys. 81 109-62.

[21] Ni Z, Liu Q, Tang K, Zheng J, Zhou J, Qin R, Gao Z, Yu D, Lu J 2012 Tunable bandgap in silicene and germanene Nano Lett. 12 113-8.

[22] Drummond N D, Zólyomi V, Fal'ko V I 2012 Electrically tunable band gap in silicene Phys. Rev. 
B 85075423.

[23] Lew Yan Voon L C, Sandberg E, Aga R S, Farajian A A 2010 Hydrogen compounds of group-iv nanosheets Appl. Phys. Lett. 97163114.

[24] Houssa M, Scalise E, Sankaran K, Pourtois G, Afanas'ev V V, Stesmans A 2011 Electronic properties of hydrogenated silicene and germanene Appl. Phys. Lett. 98223107.

[25] Wang X Q, Li H D, Wang J T 2012 Induced ferromagnetism in one-side semihydrogenated silicene and germanene Phys. Chem. Chem. Phys. 14 3031-6.

[26] Zhang C-w, Yan S-s 2012 First-principles study of ferromagnetism in two-dimensional silicene with hydrogenation J. Phys. Chem. C 116 4163-6.

[27] Gao N, Zheng W T, Jiang Q 2012 Density functional theory calculations for two-dimensional silicene with halogen functionalization Phys. Chem. Chem. Phys. 14 257-61.

[28] Ding Y, Wang Y 2012 Electronic structures of silicene fluoride and hydride Appl. Phys. Lett. 100 083102.

[29] Du Y et al. 2014 Tuning the band gap in silicene by oxidation ACS Nano 8 10019-25.

[30] Wang R, Pi X, Ni Z, Liu Y, Lin S, Xu M, Yang D 2013 Silicene oxides: Formation, structures and electronic properties Sci. Rep. 33507.

[31] Feng B, Ding Z, Meng S, Yao Y, He X, Cheng P, Chen L, Wu K 2012 Evidence of silicene in honeycomb structures of silicon on ag(111) Nano Lett. 12 3507-11.

[32] Lin C-L, Arafune R, Kawahara K, Tsukahara N, Minamitani E, Kim Y, Takagi N, Kawai M 2012 Structure of silicene grown on Ag(111) Appl. Phys. Express 5045802.

[33] Jamgotchian H, Colignon Y, Hamzaoui N, Ealet B, Hoarau J Y, Aufray B, Biberian J P 2012 Growth of silicene layers on Ag(111): Unexpected effect of the substrate temperature J. Phys.: Condens. Matter 24172001.

[34] Chiappe D, Grazianetti C, Tallarida G, Fanciulli M, Molle A 2012 Local electronic properties of corrugated silicene phases Adv. Mater. 24 5088-93.

[35] Resta A, Leoni T, Barth C, Ranguis A, Becker C, Bruhn T, Vogt P, Le Lay G 2013 Atomic structures of silicene layers grown on Ag(111): Scanning tunneling microscopy and noncontact atomic force microscopy observations Sci. Rep. 32399.

[36] Vogt P, De Padova P, Quaresima C, Avila J, Frantzeskakis E, Asensio M C, Resta A, Ealet B, Le Lay G 2012 Silicene: Compelling experimental evidence for graphenelike two-dimensional silicon Phys. Rev. Lett. 108155501.

[37] Chen L, Li H, Feng B, Ding Z, Qiu J, Cheng P, Wu K, Meng S 2013 Spontaneous symmetry breaking and dynamic phase transition in monolayer silicene Phys. Rev. Lett. 110085504.

[38] Tao L, Cinquanta E, Chiappe D, Grazianetti C, Fanciulli M, Dubey M, Molle A, Akinwande D 2015 Silicene field-effect transistors operating at room temperature Nat. Nanotechnol. 10 227-31.

[39] Radisavljevic B, Radenovic A, Brivio J, Giacometti V, Kis A 2011 Single-layer $\mathrm{MoS}_{2}$ transistors Nat. Nanotechnol. 6 147-50.

[40] Gao J, Zhang J, Liu H, Zhang Q, Zhao J 2013 Structures, mobilities, electronic and magnetic properties of point defects in silicene Nanoscale 5 9785-92.

[41] An R-L, Wang X-F, Vasilopoulos P, Liu Y-S, Chen A-B, Dong Y-J, Zhai M-X 2014 Vacancy effects on electric and thermoelectric properties of zigzag silicene nanoribbons J. Phys. Chem. C $\mathbf{1 1 8}$ 21339-46.

[42] Zha D, Chen C, Wu J, Wang M 2015 Effect of sw defect on structural and transport properties of silicene nanoribbons Int. J. Mod. Phys. B 291550061. 
[43] Li S, Wu Y, Tu Y, Wang Y, Jiang T, Liu W, Zhao Y 2015 Defects in silicene: Vacancy clusters, extended line defects, and di-adatoms Sci. Rep. 57881.

[44] Le M-Q, Nguyen D-T 2014 The role of defects in the tensile properties of silicene Appl. Phys. A 118 1437-45.

[45] Li H-p, Zhang R-q 2012 Vacancy-defect-induced diminution of thermal conductivity in silicene Europhys. Lett. 9936001.

[46] Berdiyorov G R, Peeters F M 2014 Influence of vacancy defects on the thermal stability of silicene: A reactive molecular dynamics study RSC Adv. 4 1133-7.

[47] Sahin H, Sivek J, Li S, Partoens B, Peeters F M 2013 Stone-Wales defects in silicene: Formation, stability, and reactivity of defect sites Phys. Rev. B $\mathbf{8 8} 045434$.

[48] Özçelik V O, Gurel H H, Ciraci S 2013 Self-healing of vacancy defects in single-layer graphene and silicene Phys. Rev. B 88045440.

[49] Manjanath A, Singh A K 2014 Low formation energy and kinetic barrier of Stone-Wales defect in infinite and finite silicene Chem. Phys. Lett. 592 52-5.

[50] Li R, Han Y, Dong J 2015 Substrate effects on the monovacancies of silicene: Studied from first principle methods Phys. Chem. Chem. Phys. 17 22969-76.

[51] Jamgotchian H, Colignon Y, Ealet B, Parditka B, Hoarau J-Y, Girardeaux C, Aufray B, Bibérian J-P 2014 Silicene on $\operatorname{Ag}(111)$ : Domains and local defects of the observed superstructures J. Phys.: Conf. Series 491012001.

[52] Grazianetti C, Chiappe D, Cinquanta E, Tallarida G, Fanciulli M, Molle A 2014 Exploring the morphological and electronic properties of silicene superstructures Appl. Surf. Sci. 291 109-12.

[53] Tchalala M R, Enriquez H, Yildirim H, Kara A, Mayne A J, Dujardin G, Ali M A, Oughaddou H 2014 Atomic and electronic structures of the $(\sqrt{13} \times \sqrt{13}) \mathrm{R} 13.9^{\circ}$ of silicene sheet on $\mathrm{Ag}(111)$ Appl. Surf. Sci. 303 61-6.

[54] Majzik Z et al. 2013 Combined AFM and stm measurements of a silicene sheet grown on the Ag(111) surface J. Phys.: Condens. Matter 25225301.

[55] Liu Z-L et al. 2014 Various atomic structures of monolayer silicene fabricated on Ag(111) New J. Phys. 16075006.

[56] Sone J, Yamagami T, Aoki Y, Nakatsuji K, Hirayama H 2014 Epitaxial growth of silicene on ultra-thin Ag(111) films New J. Phys. 16095004.

[57] Molle A, Chiappe D, Cinquanta E, Grazianetti C, Fanciulli M, Scalise E, van den Broek B, Houssa M 2013 Structural and chemical stabilization of the epitaxial silicene ECS Transactions 58 217-27.

[58] Gao J, Zhao J 2012 Initial geometries, interaction mechanism and high stability of silicene on Ag(111) surface Sci. Rep. 2861.

[59] Banhart F, Kotakoski J, Krasheninnikov A V 2010 Structural defects in graphene ACS Nano 5 26-41.

[60] Cahangirov S, Audiffred M, Tang P, Iacomino A, Duan W, Merino G, Rubio A 2013 Electronic structure of silicene on Ag(111): Strong hybridization effects Phys. Rev. B 88035432.

[61] Quhe R, Yuan Y, Zheng J, Wang Y, Ni Z, Shi J, Yu D, Yang J, Lu J 2014 Does the dirac cone exist in silicene on metal substrates? Sci. Rep. 45476.

[62] Wei W, Dai Y, Huang B, Whangbo M-H, Jacob T 2015 Loss of linear band dispersion and trigonal structure in silicene on Ir(111) J. Phys. Chem. Lett. 6 1065-70.

[63] Satta M, Colonna S, Flammini R, Cricenti A, Ronci F 2015 Silicon reactivity at the Ag(111) 
surface Phys. Rev. Lett. 115026102.

[64] Mills G, Jónsson H, Schenter G K 1995 Reversible work transition state theory: Application to dissociative adsorption of hydrogen Surf. Sci. 324 305-37.

[65] Kittel C, Introduction to solid state physics. John Wiley \& Sons, Inc.: Hoboken, USA, 2005.

[66] Liu Z-L et al. 2014 The fate of the $2 \sqrt{3} \times 2 \sqrt{3} \mathrm{R}\left(30^{\circ}\right)$ silicene phase on $\mathrm{Ag}(111)$ APL Mater. 2 092513.

[67] H. Jamgotchian B E, Y. Colignon, H. Maradj, J-Y. Hoarau, J-P. Biberian, B. Aufray 2015 A comprehensive study of the $(2 \sqrt{3} \times 2 \sqrt{3}) \mathrm{R} 30^{\circ}$ structure of silicene on $\mathrm{Ag}(111)$ arXiv:1412.4902

[68] Kresse G, Furthmüller J 1996 Efficient iterative schemes for ab initio total-energy calculations using a plane-wave basis set Phys. Rev. B 54 11169-86.

[69] Kresse G, Joubert D 1999 From ultrasoft pseudopotentials to the projector augmented-wave method Phys. Rev. B 59 1758-75.

[70] Perdew J P, Burke K, Ernzerhof M 1996 Generalized gradient approximation made simple Phys. Rev. Lett. 77 3865-8.

[71] Tersoff J, Hamann D R 1983 Theory and application for the scanning tunneling microscope Phys. Rev. Lett. 50 1998-2001.

[72] Xu X et al. 2014 Effects of oxygen adsorption on the surface state of epitaxial silicene on $\mathrm{Ag}(111)$ Sci. Rep. 47543.

[73] Zhuang J et al. 2015 Investigation of electron-phonon coupling in epitaxial silicene by in situ raman spectroscopy Phys. Rev. B 91161409. 